\title{
Silsesquioxanes: Recent Advancement and Novel Applications
}

\author{
Yoshiro Kaneko, ${ }^{1}$ E. Bryan Coughlin, ${ }^{2}$ Takahiro Gunji, ${ }^{3}$ Maki Itoh, ${ }^{4}$ \\ Kimihiro Matsukawa, ${ }^{5}$ and Kensuke Naka ${ }^{6}$ \\ ${ }^{1}$ Department of Chemistry, Biotechnology, and Chemical Engineering, Graduate School of Science and Engineering, \\ Kagoshima University, Kagoshima, Kagoshima 890-0065, Japan \\ ${ }^{2}$ Department of Polymer Science and Engineering, University of Massachusetts Amherst, Amherst, MA 01003, USA \\ ${ }^{3}$ Department of Pure and Applied Chemistry, Faculty of Science and Technology, Tokyo University of Science, Noda, \\ Chiba 278-8510, Japan \\ ${ }^{4}$ Electronics Solutions S\&T, Dow Corning, Ichihara, Chiba 299-0108, Japan \\ ${ }^{5}$ Electronic Material Research Division, Osaka Municipal Technical Research Institute, Osaka, Osaka 536-8553, Japan \\ ${ }^{6}$ Department of Chemistry and Materials Technology, Graduate School of Science and Technology, Kyoto Institute of Technology, \\ Kyoto, Kyoto 606-8585, Japan
}

Correspondence should be addressed to Yoshiro Kaneko, ykaneko@eng.kagoshima-u.ac.jp and Maki Itoh, maki.itoh@dowcorning.com

Received 6 November 2012; Accepted 6 November 2012

Copyright ( $) 2012$ Yoshiro Kaneko et al. This is an open access article distributed under the Creative Commons Attribution License, which permits unrestricted use, distribution, and reproduction in any medium, provided the original work is properly cited.

Among the products in silicone industry, silicone oils or elastomers are based on linear polysiloxanes mainly consisting of $\mathrm{D}\left(\mathrm{R}_{2} \mathrm{SiO}_{2 / 2}\right)$ unit, which is the predominant material in this industry mostly as polydimethylsiloxane. In contrast, silicone resins are network polymers consisting mainly of $\mathrm{T}\left(\mathrm{RSiO}_{3 / 2}\right)$ and $\mathrm{Q}\left(\mathrm{SiO}_{4 / 2}\right)$ units. Silsesquioxanes are one class of silicone resins that consist only of the $\mathrm{T}$ unit. The first commercialization of silicones started with silicone resins consisting primarily of silsesquioxanes for electrical insulation at high temperatures, in an effort to develop materials much more thermally stable and tougher than plastics but more flexible than glass [1]. Development research was started in the 1930s in Corning Glass Works and General Electric Company on the basis of academic work by Kipping. The research work at Corning Glass Works, led by Hyde, and scale-up production of the raw materials at Dow Chemical resulted in formation of Dow Corning in 1943. General Electric, where Patnode and Rochow were acting as pioneers, started commercial production of silicones in 1946 [2].

Most of the industrial silicone resin materials can be classified either into a DT type, which consists of D and T units, and an MQ type, which comprise $\mathrm{M}\left(\mathrm{R}_{3} \mathrm{SiO}_{1 / 2}\right)$ and $\mathrm{Q}$ units [3]. Silsesquioxanes could be considered as one of the DT silicone reins in which the content of the D unit is zero. In other words, $\mathrm{T}$ units without other components can form a material that we can call a silicone resin. Another characteristic of silsesquioxane is the defined structures like cages, known as polyhedral oligomeric silsesquioxanes (POSSs), which is drawing attentions both from academia and industry. Linear polymers could be characterized mostly by molecular weight and molecular weight distribution. However, the trifunctional nature of silsesquioxanes allows to form a variety of structures from oligomeric cages to ladder-like structures to three-dimensional network structures based on the ring structures. This could open up the possibility of creating unmet properties by structural control.

Silicones, including silsesquioxanes, are characterized with their high thermal/photo stability, electric insulation and so on. Compared with silica, silsesquioxanes or silicone resins can provide additional features including lower $\mathrm{k}$, flowability, flexibility, functionality, and interaction with organic molecules from the combination of siloxane backbone and organic substitution. Combination of these characteristics and the numerous possibility of structural permutations provide the unique attributes of silsesquioxane 
materials. This special issue highlights the recent significant progress in silsesquioxanes and their novel applications.

This special issue contains four review papers and eight research articles. One research article, "A synthetic route to quaternary pyridinium salt-functionalized silsesquioxanes," describes synthesis of POSS derivatives having a pendant quaternized 4-(2-ethyl)pyridyl group aiming for potential antimicrobial property. The possible benefit of using POSS is its thermal stability and the spacing of the functionality by the cubic octamers. A 4-(2-ethyl)pyridine POSS derivative was prepared by reacting the corresponding trichlorosilane either directly with cyclohexyl-substituted $\left[\mathrm{RSiO}_{3 / 2}\right]_{4}\left[\mathrm{RSi}(\mathrm{OH}) \mathrm{O}_{2 / 2}\right]_{3}$ or after converting the $\mathrm{SiOH}$ to SiOLi. Another characteristic of such defined cage structures is entrapment of gas molecules, cations, or anions. Computer simulation for the insertion reaction of various guest species for Ti-POSS, $\left[\mathrm{HTiO}_{3 / 2}\right]_{n}, n=8$ and 10 , is discussed in "A theoretical study of the insertion of atoms and ions into titanosilsequioxane (Ti-POSS) in comparison with POSS" in comparison with that for Si-POSS, exploring the similarity and difference between Ti-POSS and Si-POSS. Presence of cage compounds in products by the hydrolytic polycondensation of methyltrichlorosilane in excess water is clarified in "Characterization and some insights into the reaction chemistry of polymethylsilsesquioxane or methyl silicone resins". An experimental evidence that such cage molecules are formed by siloxane bond rearrangement from molecules of similar molecular weight rather than simple condensation of precursor molecules is presented. The presence of such cage molecules in industrial DT type silicone resins is also described. Preparation of block copolymers of methacrylates having POSS and ferrocene in the side chain by anionic polymerization is described in "Iron oxide arrays prepared from ferrocene- and silsesquioxane-containing block copolymers." The materials showed microphase-separated nanostructures, and the array is expected to be a promising catalytic material for the creation of carbon nanotube thin films.

Another form of defined structure for silsesquioxane is a ladder structure. Proven ladder structure of silsesquioxanes can be found in those synthesized by stepwise reactions. The first nonacyclic ladder silsesquioxanes with isopropyl substituent are reported in "Nonacyclic ladder silsesquioxanes and spectral features of ladder polysilsesquioxanes", as formed by the reaction of bicyclic silanol with tricyclic tetrachloride. A review paper, "Preparation of ionic silsesquioxanes with regular structures and their hybridization," describes the preparation of ladder-like polysilsesquioxanes by self-organization of ammonium salt, for which anion exchange behavior is investigated. In this review, control of conformational structures of the polysilsesquioxanes by the introduction of chiral moieties is also reported.

Various polysilsesquioxanes including hybrids aiming for specific functions are presented. Polysilsesquioxanes bearing ethoxysulfonyl groups in side chains were prepared for a proton-conductive film, by the hydrolytic polycondensation of 4-(2-methyl-3-triethoxysilylpropoxy)benzenesulfonate as discussed in "Synthesis and properties of polysilsesquioxanes having ethoxysulfonyl group as a side chain." Polymethylsilsesquioxane-based organic-inorganic hybrid materials for gate insulating layer in organic thin film transistors (TFTs) are reviewed in "Polysilsesquioxanes for gate insulating materials of organic thin-film transistors," referring to the possibility for flexible TFTs. Water-soluble polysilsesquioxanes, including hydroxyl-functional and cationic silsesquioxanes, are reviewed including the hybrids of $\mathrm{SiO}_{1.5} / \mathrm{SiO}_{2}$ and $\mathrm{RSiO}_{1.5} / \mathrm{TiO}_{2}$ and stimuli-responsive materials in "Design and synthesis of functional silsesquioxane-based hybrids by hydrolytic condensation of bulky triethoxysilanes." Hybrid nanoparticles with other metal oxides are also reported for poly-3-aminopropyl-silsiesquioxane and zirconia having the estimated refractive index of 1.66, which can form highly transparent water dispersion as discussed in "Sol-gel preparation of highly water-dispersible silsesquioxane/zirconium oxide hybrid nanoparticles."

The last set of materials is so-called bridged silsesquioxanes. Ethylene-bridged polysilsesquioxane gels with hierarchical macropores and mesopores are prepared by combining the micellar templating in nanometer-scale with the polymerization-induced phase separation in micrometerscale for well defined mesoporous structures as described in "Evolution of mesopores in monolithic macroporous ethylenebridged polysilsesquioxane gels incorporated with nonionic surfactant." Synthesis and properties of oligo-thiophene bridged polysilsesquioxanes are reviewed in "Synthesis of a novel family of polysilsesquioxanes having oligothiophenes with well-defined structures." The polymers anchored on $\mathrm{SiO}_{2}$ or ITO substrates showed excellent mechanical hardness based on the three-dimensional siloxane network structure with chemical linkage between the polymer and the surface of the metal-oxide substrates.

\section{Acknowledgment}

The editors are grateful to the contributing authors and reviewers for their efforts in compiling this special issue.

\section{Yoshiro Kaneko \\ E. Bryan Coughlin \\ Takahiro Gunji \\ Maki Itoh \\ Kimihiro Matsukawa \\ Kensuke Naka}

\section{References}

[1] E. L. Warrick, "Years of firsts," in The Recollections of a Dow Corning Pioneer, chapter 1, pp. 1-45, McGraw-Hill, New York, NY, USA, 1990.

[2] E. G. Rochow, Silicon and Silicones, Springer, Berlin, Germany, 1987.

[3] R. H. Baney, M. Itoh, A. Sakakibara, and T. Suzuki, "Silsesquioxanes," Chemical Reviews, vol. 95, no. 5, pp. 14091430, 1995. 

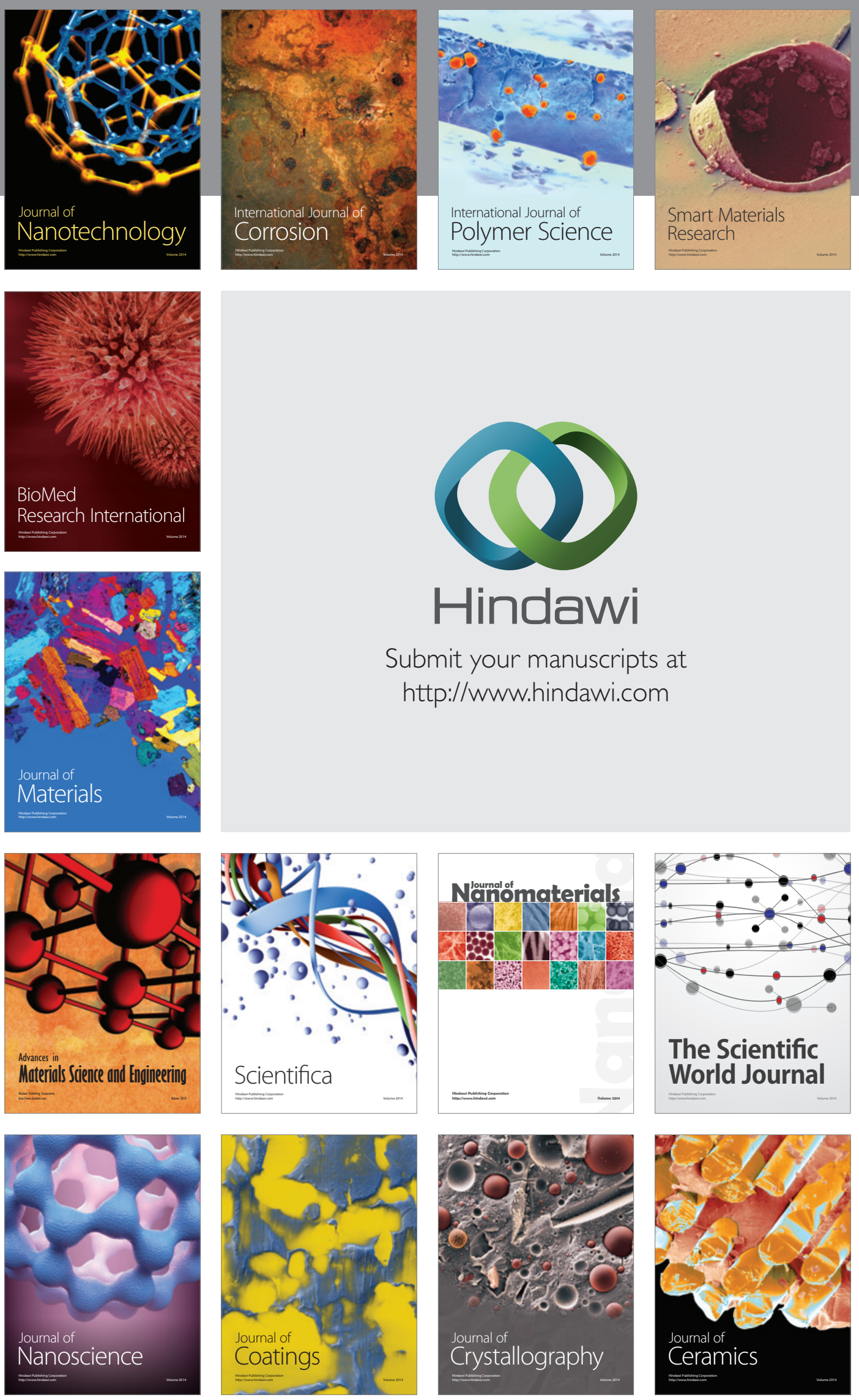

The Scientific World Journal

Submit your manuscripts at

http://www.hindawi.com

\section{World Journal}

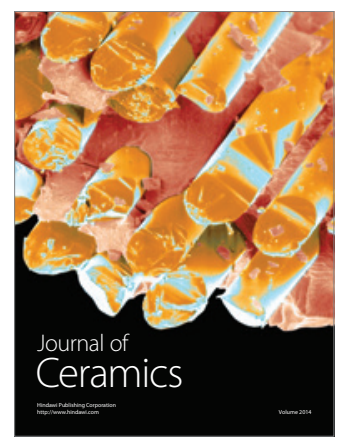

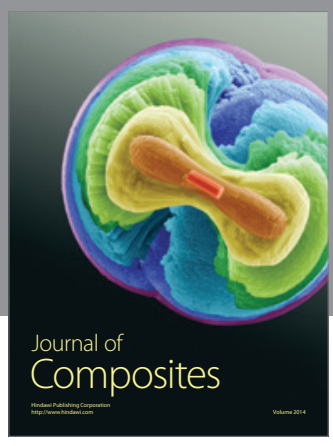
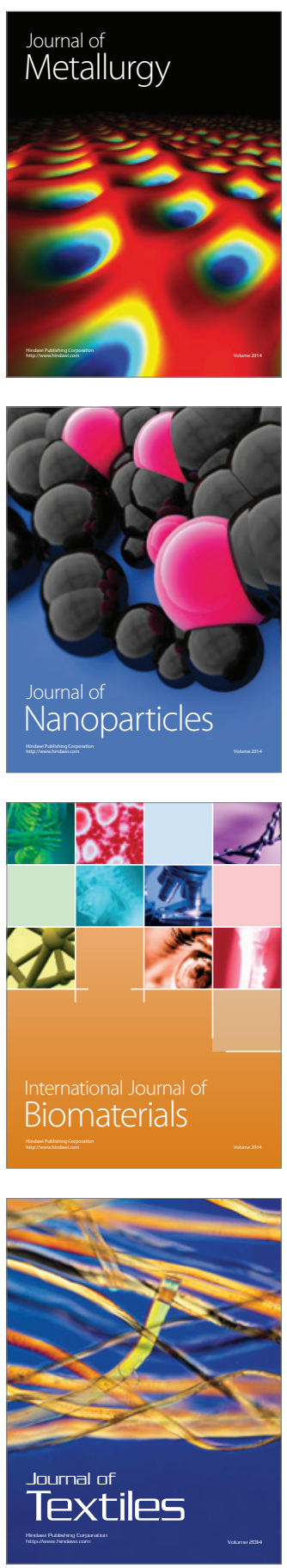